\section{Genetics of progressive hearing loss: a link between hearing impairment and dysfunction of mechanosensory hair cells}

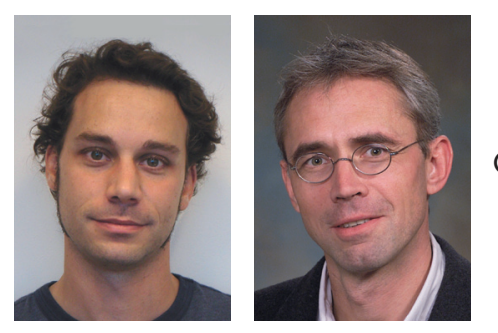

\author{
"In order to define and treat the \\ pathophysiological changes that \\ cause hearing loss, it will be essential to \\ define the function of the genes that \\ are linked to auditory impairment."
}

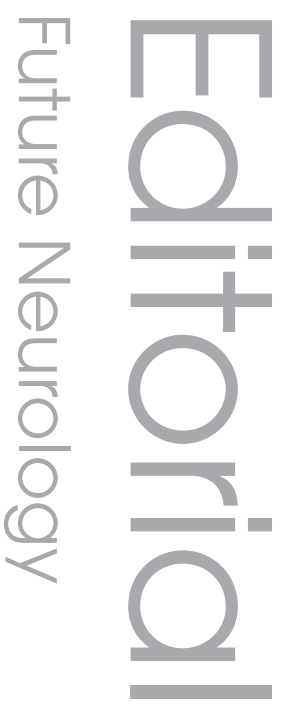

Nicolas Grilleł ${ }^{\dagger}$ \& Ulrich Müller

†Author for correspondence: Department of Cell Biology, The Scripps Research Institute, La Jolla,

CA, 92037, USA = Tel.. +1 8587847288 = Fax: +1 8587847299 m grillet@scripps.edu

Hearing loss is the most common form of sensory impairment that affects the population in developed countries. Although it is not widely appreciated, a large number of children (1-2 per $1000)$ are born deaf. However, most of us know from personal experience in our circle of friends and family that the disease frequently affects elderly people $[1,2]$. Hearing loss is a complex disease that can be subdivided into categories depending on several criteria: the part of the ear that is affected (external and middle ear in conductive hearing loss; the inner ear in sensorineural forms of the disease); the age of disease onset (congenital, pre-/post-lingual, age-related and presbycusis); its degree of severity (moderate, mild, severe and profound); the affected frequencies (low: <500 Hz; middle: 500-2000 Hz; high: $>2000 \mathrm{~Hz}$ ); and the association with other symptoms, such as vision loss (syndromic) or not (nonsyndromic).

\section{6...LOXHDI, PJVK and MYO3A are}

expressed in the inner ear in hair cells, the mechanosensory cells that convert sound waves and head movements into electrical signals to provide our sense of hearing and balance."

Hearing impairment can be triggered by environmental factors, such as exposure to excessive noise, chemicals and certain medications that as a side effect perturb auditory function [3]. Many forms of hearing loss are of genetic origin, and genetic predisposition is also thought to be an important factor in the development of noise- and age-dependent forms of the disease. Approximately $15 \%$ of all genetic forms of deafness are inherited as dominant traits, while $80 \%$ are transmitted recessively; the remaining patients carry mutations in the sex chromosomes or even in the genome of mitochondria - the organelles within our cells that generate energy $[1,2]$. While recessive deafness generally leads to congenital and profound hearing loss, dominant deafness tends to be lateonset and progressive. To date, approximately 50 genes have been linked to hearing loss, but many more genes are thought to be involved [101]. These genes encode proteins with various functions, such as transcription factors, adhesion receptors, cytoskeletal components, connexins, ion channels and synaptic proteins.

In order to define and treat the pathophysiological changes that cause hearing loss, it will be essential to define the function of the genes that are linked to auditory impairment. As agerelated hearing loss is a fast-growing problem, owing to the increase in life expectancy, it will also be important to determine how interactions between genetic and environmental factors come into play to determine disease onset and progression. Progress in genomic research has accelerated the pace of auditory research by providing novel tools for the identification of genetic changes that cause hearing loss. The identification of the causative mutations has been greatly aided by the elucidation of the human genome. However, it is still a difficult task to identify the causative mutations and requires access to clinical data and DNA samples from members of families that are afflicted with genetic forms of the disease. An alternative approach to establishing a connection between genetic changes and hearing loss is to focus on vast numbers of affected and nonaffected humans and scrutinize 
the entire genome for features shared between affected individuals. This is the aim of the recent genome-wide association studies. One of these studies has already focused on the identification of genetic alterations that confer an increased risk for the development of age-related hearing loss. This study identified a highly significant SNP in the gene coding a metabotropic glutamate receptor (GRM7) [4]. With the availability of affordable next-generation sequencing technologies, future studies will probably identify additional significant SNPs and susceptibility genes by using better genomic coverage and a higher number of patients.

\section{“...defects in hair cell function ... might be the cause of progressive forms of hearing loss in humans."}

There is no doubt that genome-wide association studies are an instrumental tool in the identification of polymorphisms that are most commonly associated with hearing loss in humans. However, some mutations are rare and need to be identified by alternative strategies. Genetically tractable animal models are particularly important in this regard. Studies carried out over the last few years have indeed demonstrated that the mouse is a particularly useful model to study the human disease. The inner ear of mice and humans share many structural and functional similarities and the genetic program that controls the development and function of the auditory sense organ appears to be quite conserved. Capitalizing on these premises, several laboratories have demonstrated that mutations in the same genes frequently lead to hearing loss in both humans and mice. For examples, studies focusing on mice in large breeding colonies that are maintained for research purposes have led to the identification of spontaneous mutations with defects in hearing function. These mice can be detected in the mouse colonies with relative ease because the inner ear also contains sensors for head movement, meaning that affected mice are frequently hyperactive and circle. The identification of the mutations that cause inner-ear dysfunction in these mice has led to the identification of several genes that are also linked to hearing loss in humans $[5,6]$.

The identification and analysis of sporadic mutations in mouse colonies is time consuming because the mutations are rare and this has led to only a limited number of animal models for auditory research. New strategies have therefore been developed to increase the repertoire of mouse strains with defined auditory phenotypes by treating mice with mutagens. The alkylating agent $N$-ethyl- $N$-nitrosourea (ENU) has been chosen most commonly because it is highly effective and introduces point mutations randomly. Since many human genetic diseases are caused by point mutations, some of the mice that are obtained by ENU mutagenesis are expected to be valuable animal models for the diseases. The first systematic ENU mutagenesis to identify mouse lines that are afflicted with recessive forms of hearing loss was carried out in our laboratory [7]. We chemically induced mutations randomly in the genome of male gametes, bred the mice for several generations and identified those with heritable hearing loss.

One of the more than 20 lines from our screen, termed samba, contains a mutation that affects a previously uncharacterized gene termed lipoxygenase homology domain 1 (LOXHD1) [8]. We resolved the complete gene structure of Loxhd 1 and demonstrated that it is expressed in the inner ear of mice. LOXHD1 encodes a protein of 2068 amino acids with an unusual structure. It consists of 15 so-called polycystin lipoxygenase $\alpha$-toxin (PLAT) domains. A single PLAT domain consists of 120 amino acids and is present in proteins of diverse function, such as lipoxygenases, pancreatic lipase, the rab6-interacting protein 1 and the $\alpha$-toxin of Clostridium perfringens [9-12]. While the function of PLAT domains is unknown, previous works have suggested that PLAT domains are able to interact with the plasma membrane and, in some cases, can also interact with other proteins [13]. Crystallographic studies have demonstrated that PLAT domains form a $\beta$-sandwich consisting of two sheets, each of four strands, creating a highly hydrophobic pocket. The amino acids in the pocket are highly conserved in PLAT domains and one of them is mutated in samba mice, changing it from a hydrophobic residue (isoleucine 1342) into a polar one (asparagine) [8]. This is predicted to be deleterious for the proper folding of the PLAT domain, and may affect the overall structure of the entire LOXHD1 protein.

Based on our findings in mice, we searched for mutations in human families segregating autosomal recessive hearing loss. We linked a genomic region on human chromosome 18, which includes the LOXHD1 gene, to hearing impairment in a consanguineous Iranian family. Subsequent analysis of genomic DNA by sequencing revealed a missense mutation in LOXHD1 that is homozygous only in affected 
family members [8]. The mutation is predicted to truncate LOXHD1 after the fifth PLAT domain. Affected members of the family show preferential hearing loss in the mid- to high-frequency range that can already be detected in childhood and is aggravated during aging, finally leading to complete deafness. Interestingly, only three genes have so far been linked to progressive forms of hearing loss that are inherited as recessive traits. We have linked mutations in a gene termed pejvakin $(P J V K)$ to this form of hearing loss and others established a link between myosin 3a $(M Y O 3 A)$ and the disease [7,14]. Since the $L O X H D 1$ gene is very large - it spans more than $160 \mathrm{kB}$ - it will be important to determine whether other $L O X H D 1$ mutations might cause other forms of disease, such as congenital or age-related hearing impairment.

"...age-related hearing loss is a

fast-growing problem owing to the increase in life expectancy..."

Interestingly, LOXHD1, PJVK and MYO3A are expressed in the inner ear in hair cells, the mechanosensory cells that convert sound waves and head movements into electrical signals to provide our sense of hearing and balance. These findings suggest that defects in hair cell function more generally might be the cause of

\section{Bibliography}

Papers of special note have been highlighted as:

- of interest

-" of considerable interest

1. Hildebrand MS, Newton SS, Gubbels SP et al.: Advances in molecular and cellular therapies for hearing loss. Mol. Ther. 16(2), 224-236 (2008).

2. Hilgert N, Smith RJ, Van Camp G: Function and expression pattern of nonsyndromic deafness genes. Curr. Mol. Med. 9(5), 546-564 (2009).

3. Van Eyken E, Van Camp G, Van Laer L: The complexity of age-related hearing impairment: contributing environmental and genetic factors. Audiol. Neurootol. 12(6), 345-358 (2007).

4. Friedman RA, Van Laer L, Huentelman MJ et al: Grm 7 variants confer susceptibility to age-related hearing impairment. Hum. Mol. Genet. 18(4), 785-796 (2009).

- First large-scale, genome-wide association study for age-related hearing loss and identification of a highly significant associated SNP in the GRM7 gene.

progressive forms of hearing loss in humans. The mechanically sensitive organelle of hair cells is the so-called hair bundle, an array of actin-rich protrusions that emanate from the apical hair cell surface. Mechanosensors with actin-rich protrusions are found in the highly developed Chordates but not in the evolutionarily divergent Ecdysozoa (insect, nematode). LOXHD1 is found in the former but not the latter, raising the possibility that LOXHD1 is an evolutionary change that has enabled the emergence of actin-based mechanosensory structures.

In summary, our findings demonstrate that the mouse is a powerful instrument for auditory research, enabling the discovery of genes that cause hearing loss in humans and providing model systems to study disease mechanisms. One of the next big challenges is to utilize these mouse lines for the development of therapeutic strategies towards the treatment of hearing loss.

\section{Financial \& competing interests disclosure \\ The authors have no relevant affiliations or financial involvement with any organization or entity with a financial interest in or financial conflict with the sub- ject matter or materials discussed in the manuscript. This includes employment, consultancies, honoraria, stock ownership or options, expert testimony, grants or patents received or pending, or royalties. \\ No writing assistance was utilized in the production of this manuscript.}

5. Noben-Trauth K, Johnson KR: Inheritance patterns of progressive hearing loss in laboratory strains of mice. Brain Res. 1277, 42-51 (2009).

6. Brown SD, Hardisty-Hughes RE, Mburu P: Quiet as a mouse: dissecting the molecular and genetic basis of hearing. Nat. Rev. Genet. 9(4), 277-290 (2008).

7. Schwander M, Sczaniecka A, Grillet $\mathrm{N}$ et al.: A forward genetics screen in mice identifies recessive deafness traits and reveals that pejvakin is essential for outer hair cell function. J. Neurosci. 27(9), 2163-2175 (2007).

- First forward recessive screen for hearing loss in mice and identication of mutations in the gene PJVK as the cause of deafness in humans and mice.

8. Grillet N, Schwander M, Hildebrand MS et al:: Mutations in loxhd1, an evolutionarily conserved stereociliary protein, disrupt hair cell function in mice and cause progressive hearing loss in humans. Am. J. Hum. Genet. 85(3), 328-337 (2009).
- Identification of mutations in the uncharacterized $L O X H D 1$ gene as causing deafness in mice and humans. In DFNB77 patients, the deafness is progressive and age-related. In mice, $L O X H D 1$ is demonstrated to be expressed in the hair cell stereocilia - the organelle of mechanotransduction.

9. Gillmor SA, Villasenor A, Fletterick R, Sigal E, Browner MF: The structure of mammalian 15-lipoxygenase reveals similarity to the lipases and the determinants of substrate specificity. Nat. Struct. Biol. 4(12), 1003-1009 (1997).

10. Minor W, Steczko J, Stec B et al.: Crystal structure of soybean lipoxygenase L-1 at $1.4 \mathrm{~A}$ resolution. Biochemistry 35(33), 10687-10701 (1996).

11. Recacha $\mathrm{R}$, Boulet $\mathrm{A}$, Jollivet $\mathrm{F}$ et al.: Structural basis for recruitment of rab6-interacting protein 1 to golgi via a run domain. Structure 17(1), 21-30 (2009). 


\section{Editorial Grillet \& Müller}

12. Van Tilbeurgh H, Egloff MP, Martinez C, Rugani N, Verger R, Cambillau C: Interfacial activation of the lipase-procolipase complex by mixed micelles revealed by $\mathrm{x}$-ray crystallography. Nature 362 (6423), 814-820 (1993).

13. Bateman A, Sandford R: The plat domain: a new piece in the PKD1 puzzle. Curr. Biol. 9(16), R588-R590 (1999).
14. Walsh T, Walsh V, Vreugde S et al.: From flies' eyes to our ears: Mutations in a human class iii myosin cause progressive nonsyndromic hearing loss DFNB30. Proc. Natl Acad. Sci. USA 99(11), 7518-7523 (2002).

\section{Website}

101. The Hereditary Hearing Loss Homepage http://webh01.ua.ac.be/hhh/

\section{Affiliations}

- Ulrich Müller

Department of Cell Biology,

The Scripps Research Institute, La Jolla,

CA, 92037, USA

Tel.: +18587847288

Fax: +18587847299

umueller@scripps.edu

- Nicolas Grillet

Department of Cell Biology,

The Scripps Research Institute, La Jolla, CA, 92037, USA

Tel.: +18587847288

Fax: +18587847299

grillet@scripps.edu 